\title{
ESTADO ECOLÓGICO DE LA CUENCA BAJA DEL ARROYO SAN BERNARDO, SIERRA DE LA VENTANA, ARGENTINA
}

\author{
ECOLOGICAL CONDITIONS OF THE SAN BERNARDO \\ STREAM'S LOW BASIN, SIERRA DE LA \\ VENTANA, ARGENTINA
}

\author{
Antonela Volonté \\ Alicia M. Campo ${ }^{2}$ \\ Verónica $\mathrm{Gil}^{3}$
}

Universidad Nacional del Sur, Argentina

\section{RESUMEN}

El estado ecológico de un río es aquella medida de calidad en la estructura y funcionamiento del ecosistema acuático. Los procesos geomorfológicos determinan una gran variedad de hábitats, favoreciendo la biodiversidad y productividad de los ecosistemas acuáticos y ribereños. Los indicadores hidrogeomorfológicos son claves para determinar el estado ecológico de los ríos. La dinámica hidrológica garantiza la biodiversidad, la calidad ambiental y la protección de todos los elementos del sistema y sus relaciones. El objetivo de este trabajo es conocer el estado ecológico de un tramo en la cuenca baja del arroyo San Bernardo, para lo cual se utilizó el Índice Hidrogeomorfológico

1 Lic. Antonela Volonté, Universidad Nacional del Sur, 12 de Octubre y San Juan, Bahía Blanca. Correo electrónico: antonela.volonte@uns.edu.ar

2 Dra. Alicia M. Campo, Universidad Nacional del Sur, 12 de Octubre y San Juan, Bahía Blanca. CONICET. Correo electrónico: amcampo@uns.edu.ar

3 Dra. Verónica Gil, Universidad Nacional del Sur, 12 de Octubre y San Juan, Bahía Blanca. CONICET. Correo electrónico: verogil@uns.edu.ar 
Antonela Volonté, Alicia M. Campo, Verónica Gil. Ecological conditions of the

San Bernardo stream's low basin, Sierra de la Ventana, Argentina

(IHG). Los resultados mostraron que el tramo analizado presenta una calidad buena e indica que la calidad funcional del sistema, del cauce y de las riberas se encuentran dentro de los límites aceptables, es decir, que las intervenciones antropogénicas no han impactado en la naturalidad del tramo.

Palabras clave: estado ecológico, arroyo San Bernardo, Índice Hidrogeomorfológico, geomorfología fluvial.

\begin{abstract}
The ecological status of a river is the measure of quality in the structure and functioning of the aquatic ecosystem. Geomorphological processes determine a variety of habitats, thus promoting biodiversity and productivity of aquatic and coastal ecosystems. The hydrogeomorphological indicators are key to determining the ecological status of rivers. The hydrological dynamics ensures biodiversity, environmental quality and protection of all system elements and their relationships. The aim of this study was to determine the ecological status of a section in the lower San Bernardo stream watershed, for which the hydrogeomorphological index (IHG) was used. The results showed that the section used has good quality. This is important because it indicates that the functional quality of the system, the quality of the channel, and the bank is within the acceptable limits. This means that the anthropogenic interventions have not impacted the naturalness of the section.
\end{abstract}

Keywords: ecological status, San Bernardo stream, Hydrogeomorphological Index, fluvial geomorphology

\title{
Introducción
}

Los ríos han sido siempre centro de atención en los estudios de geografía física sin embargo, el reconocimiento de su significado como agente geomorfológico es relativamente reciente. Desde la geomorfología, los ríos son agentes de erosión y de transporte, su eficacia depende de la energía de la corriente y de la cantidad de sedimentos transportados. Los rasgos dinámicos de las cuencas, explícitos en los procesos, flujos, sedimentos y formas de los canales de drenaje y redes, cambian constantemente (García, Gómez y Ortigosa., 1987). Los cauces muestran gran variabilidad en poco espacio y en cada tramo suelen haber características hídricas, vegetales y sedimentológicas diferentes.

El estado ecológico de un río es una medida de calidad de la estructura y funcionamiento del sistema acuático y se considera que un buen estado ecológico es aquel en el que las comunidades biológicas son iguales o muy próximas a las que se encontrarían en condiciones inalteradas. Esto está en estrecha relación con el caudal ecológico. En estas circunstancias las condiciones físico-químicas y las condiciones del medio acuático deben permitir el correcto desarrollo de las comunidades vivas (Martínez, Correcher, Piñón, Martínez y Pujante., 2004). Conocer el estado ecológico 
de un río es un elemento esencial en la gestión dado que refleja la calidad del agua y del medio acuático relacionando distintas variables.

A los efectos de realizar un diagnóstico que detecte problemas futuros se utilizan distintos indicadores que permiten valorar el estado ecológico del espacio fluvial. Los resultados sirven para planificar acciones, seleccionar los tramos con mayores problemas de conservación y tomar decisiones en cuanto al manejo de la cuenca. La calidad geomorfológica se evalúa usando una serie de parámetros que facilitan la descripción de manera genérica de las características de cada tramo o sub-tramo. Se trata esencialmente del grado de sinuosidad, de la pendiente del río, de las variaciones en el ancho y profundidad del canal y de la forma del valle fluvial.

La dinámica fluvial, es clave tanto del funcionamiento como del valor ecológico, paisajístico y ambiental de los sistemas fluviales. Por lo tanto, los indicadores hidrogeomorfológicos son importantes para determinar el estado ecológico de los ríos. Si se valora a la cuenca como un ecosistema y un corredor ambiental se debe proteger ante todo su dinámica hidrogeomorfológica porque esta es la que va a garantizar la biodiversidad, la calidad ambiental y la protección de todos los elementos del sistema y sus relaciones. Sólo las cuencas que mantienen una buena calidad pueden funcionar correctamente como un sistema (Ollero Ojeda, 2009; Ordeix, Camprodom y Guixe, 2012).

El arroyo San Bernardo forma parte de una de las subcuencas más importantes del río Sauce Grande al que confluye en cercanías de la localidad de Sierra de la Ventana, Buenos Aires, Argentina. Dicha cuenca presenta una dinámica torrencial, generándose crecidas intensas cuando ocurren eventos meteorológicos extremos. Estas crecidas ocasionan la inundación de sectores urbanos aledaños al curso principal, corte temporario de accesos a la localidad, evacuación de personas y pérdidas tanto humanas como económicas. Es importante tener en cuenta que toda reactivación en la dinámica fluvial de un sector antiguamente móvil pero actualmente estabilizado se traduce a corto plazo en una reactivación de la dinámica ecológica y por lo tanto, en un incremento de la biodiversidad y de la calidad de los ecosistemas acuáticos. Es por ello que el objetivo de este trabajo es conocer el estado ecológico en un tramo de la cuenca baja del arroyo San Bernardo utilizando el Índice Hidrogeomorfológico (IHG) propuesto por Ollero Ojeda (2009). 


\section{Área de estudio}

En el suroeste de la provincia de Buenos Aires (Argentina) se desarrolla el Sistema de Ventania. Es una unidad orográfica formada por un conjunto de elevaciones con sentido noroeste-sureste. El afloramiento rocoso tiene $175 \mathrm{~km}$ de longitud y alcanza un ancho máximo de $50 \mathrm{~km}$ (fig. 1). El valle del río Sauce Grande separa los cordones occidentales y orientales en el sector sur. El sector occidental comprende las Sierras de Puán, Curamalal, Bravard y Ventana mientras que la oriental está formada por las Sierras de las Tunas y de Pillahuincó. Las mayores alturas se encuentran en el sector occidental y oscilan entre $900 \mathrm{~m}$ y $1.200 \mathrm{~m}$ mientras que en el área oriental las cumbres varían entre 600 m y 750 m (Sellés, 2001).

Las sierras poseen numerosos valles transversales. La cuenca del arroyo San Bernardo se desarrolla sobre las laderas orientales de la Sierra de la Ventana. Allí se encuentra el punto más alto del sistema, el cerro Tres Picos $(1.247 \mathrm{~m})$ lugar donde nace el arroyo San Bernardo. Las rocas que afloran en la cuenca son predominantemente cuarcitas del Grupo Ventana (Harrington, 1947; Sellés, 2001).

Climáticamente se observa la presencia de veranos e inviernos térmicos bien diferenciados y estaciones intermedias muy similares en sus valores. Las localidades que se encuentran próximas al Sistema de Ventania presentan los valores medios más bajos del suroeste bonaerense mientras que las localidades que se encuentran al este presentan valores superiores. Las estaciones intermedias registran valores que oscilan en torno a los $14{ }^{\circ} \mathrm{C}$ siendo algo menores en los límites norte y este. En las localidades próximas a los cursos de agua se atenúan las temperaturas extremas al incorporar humedad al ambiente.

Las precipitaciones en el suroeste bonaerense varían espacialmente y guardan relación con la continentalidad, exposición a los flujos dominantes, orientación de la costa y las corrientes oceánicas. La continentalidad afecta a la región observándose una disminución de las precipitaciones de noreste a suroeste. En el oeste y sur de la región Bahía Blanca cuenta con $685 \mathrm{~mm}$ anuales e Hilario Ascasubi con $542 \mathrm{~mm}$ (Campo de Ferreras, Capelli de Steffens y Diez, 2004; Zapperi, Casado, Gil y Campo, 2006 y Zapperi, Ramos, Gil y Campo, 2007). Las variaciones temporales y espaciales de las precipitaciones en laderas opuestas del sistema de Ventania fueron estudiadas por Gil (2009) y Gentili, Campo y Gil (2011) quienes comprobaron que existe una importante variación espacial de las precipitaciones en el valle interserrano. 
La cuenca del arroyo San Bernardo tiene una superficie de $83 \mathrm{~km}^{2}$ es una sub-cuenca del río Sauce Grande y confluye en cercanías de la localidad turística de Sierra de la Ventana; su afluente principal es el arroyo San Diego. En el área de la cuenca se observan espacios de uso rural sobre los valles y de uso urbano en las márgenes. El caudal medio oscila entre 0,3 y $0,5 \mathrm{~m}^{3} / \mathrm{s}$ y en las crecidas ordinarias asciende a $5 \mathrm{~m}^{3} / \mathrm{s}$ aproximadamente. Con respecto a la capacidad de carga presenta sedimentos con diámetros que oscilan entre $6 \mathrm{y}$ $18 \mathrm{~cm}$, hallándose bloques de $60 \mathrm{~cm}$ (Campo, Volonté y Gil, 2014).

Figura 1. Localización del área de estudio

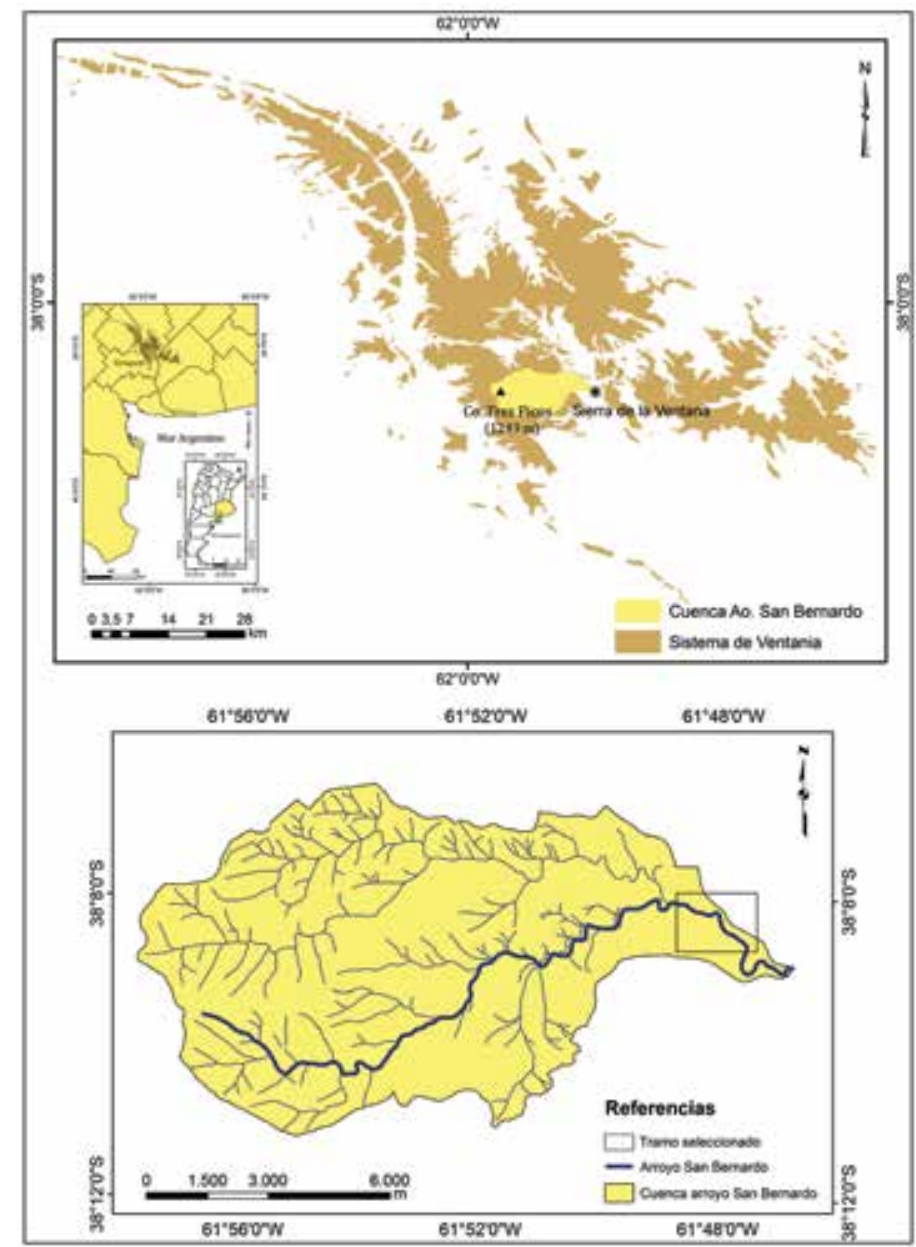

Fuente: elaborado por Volonté (2014). 
La vegetación predominante del piedemonte serrano es el pastizal de Stipa sp. y asociado a los valles se encuentra la paja colorada (Paspalum quadrifarium). En los sitios más húmedos la especie representativa es Cortaderia selloana (Matteucci, 2012). Volonté, Duval, Gil y Campo (2013) a través de la realización de transectas de vegetación identificaron 12 especies herbáceas y 1 especie arbórea como las representativas del área de ribera. Las predominantes fueron: Cortaderia selloana y Cynodon dactylon mientras que las especies con menor cantidad de ejemplares fueron: Populus spp., Galium broterianum y Carduus thoermeri. Las especies Cortaderia selloana y Cynodon dactylon se encuentran más cerca del curso de agua dado que requieren de más humedad mientras que las especies como Taraxacum officinale se encuentran en los límites de las transectas porque sus requerimientos de humedad son menores.

\section{Materiales y métodos}

La valoración de un sistema fluvial puede abordarse desde diferentes metodologías. En este trabajo se utiliza el Índice Hidrogeomorfológico (IHG) propuesto por Ollero (2009). Este índice fue creado con el objetivo de identificar y reducir los problemas ambientales de los sistemas fluviales para mejorar y conservar su funcionalidad y naturalidad, así como destacar sus valores hidrogeomorfológicos. El índice consta de tres bloques: calidad funcional del sistema fluvial (Bloque I), calidad del cauce (Bloque II) y calidad de las riberas (Bloque III). Cada uno de ellos considera a su vez tres parámetros. Cada uno de los parámetros posee un valor inicial de 10 puntos indicando la situación natural ideal del ambiente. En el caso que se observen impactos o presiones sobre el ambiente se van restando puntos (tabla 1). El índice puede presentarse como una ficha única que reúna los nueve parámetros o se puede utilizar para valorar solamente la funcionalidad del sistema, la calidad del cauce o la calidad de las riberas. En este trabajo se consideraron la totalidad de los parámetros propuestos. 
Tabla 1. Valoración integral e individual del Índice Hidrogeomorfológico

\begin{tabular}{|c|c|}
\hline \multicolumn{2}{|c|}{ Valoración integral } \\
\hline Puntos obtenidos & Calidad hidrogeomorfológica del sistema \\
\hline $75-90$ & Muy buena \\
\hline $60-74$ & Buena \\
\hline $42-69$ & Moderada \\
\hline $21-41$ & Deficiente \\
\hline $0-20$ & Muy mala \\
\hline \multicolumn{2}{|c|}{ Valoración individual } \\
\hline $25-30$ & Muy buena \\
\hline $20-24$ & Buena \\
\hline $14-19$ & Moderada \\
\hline $13-7$ & Deficiente \\
\hline $0-6$ & Muy mala \\
\hline
\end{tabular}

Fuente: elaborado por Volonté (2014) sobre la base de Ollero (2009).

Para la aplicación del índice se seleccionó un tramo en la cuenca baja del arroyo San Bernardo (fig. 2). La escala de observación ideal para la implementación de este índice es para los tramos inferiores al kilómetro, en el caso del tramo seleccionado la extensión es $1.071 \mathrm{~m}$ señalado entre barras en la figura.

Figura 2. Tramo seleccionado para la aplicación del Índice Hidrogeomorfológico

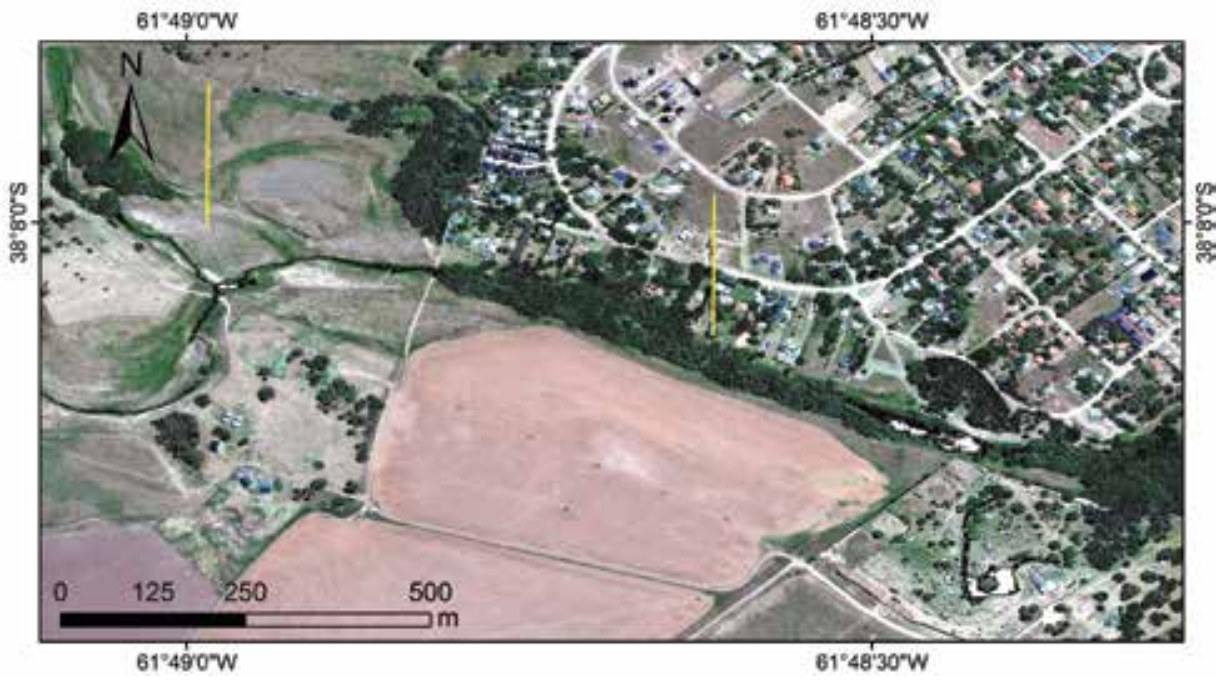

Fuente: elaborado por Volonté (2014) sobre imagen del Google Earth ${ }^{\circledR}$. 


\section{Resultados}

\section{Bloque I. Calidad funcional del sistema fluvial}

Para evaluar la calidad funcional del sistema fluvial se debe considerar el caudal y el espacio inundable. La calidad funcional para el tramo de estudio se obtuvo a partir de tres parámetros: la naturalidad del régimen de caudal (p1), la disponibilidad y movilidad de sedimentos (p2) y la funcionalidad de la llanura de inundación (p3).

Para calificar ( $\mathrm{p} 1)$ se consideró que en la mayor parte del tramo, la cantidad de caudal circulante responda a la dinámica natural, lo cual deja de suceder en los procesos extremos. La presencia de un dique de pequeñas dimensiones no modifica la cantidad de caudal circulante y el arroyo presenta una dinámica natural. Por otra parte, la vegetación ribereña y las especies arbóreas de gran porte actúan como diques naturales, retienen el agua y magnifican las crecidas (fig. 3).

La cuenca del arroyo San Bernardo posee registros de aforo para el periodo 1938-1943. El caudal medio para ese periodo fue de $0,391 \mathrm{~m}^{3} / \mathrm{s}$. Debido a la ausencia de datos se utilizó la técnica de aforo con molinete hidrométrico, se establecieron secciones y se realizaron mediciones del perímetro mojado, área de la sección transversal y velocidad de la corriente de agua a diferentes profundidades. Se realizaron cuatro mediciones que dieron como resultado un caudal medio de $0,259 \mathrm{~m}^{3} / \mathrm{s}$.

Con respecto a la disponibilidad y movilidad de los sedimentos (p2) si bien el caudal llega al sector en el cual se aplica el índice sin ningún tipo de retención antropogénica y el sistema fluvial puede ejercer la función de transporte, la presencia de especies herbáceas retienen sedimentos finos en las crecidas ordinarias. 
Figura 3. Naturalidad del régimen de caudal
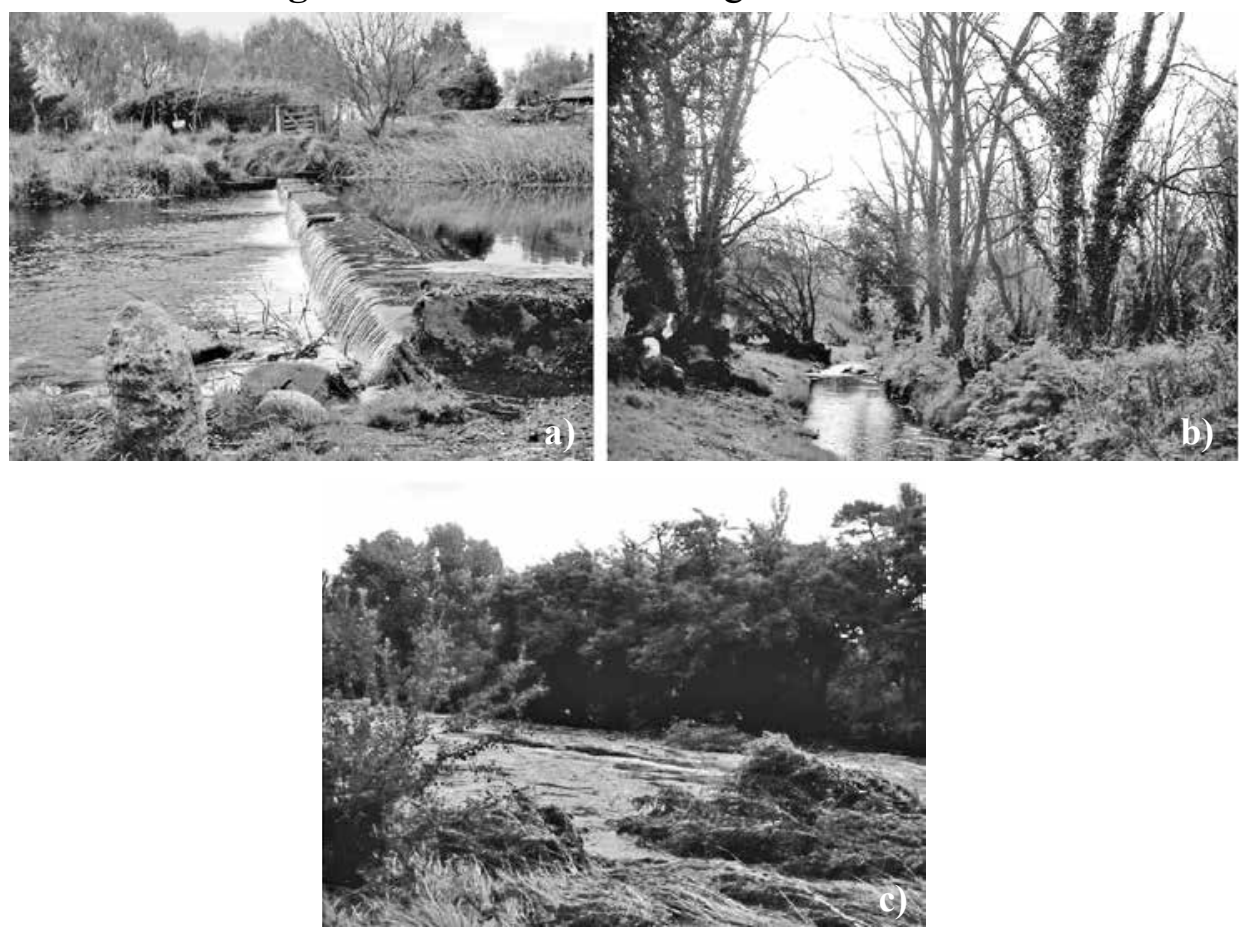

Fuente: Volonté (2014).

En la fotografía a) se puede observar el dique de pequeñas dimensiones mientras que en la fotografía b) se observa la vegetación que se encuentra sobre las márgenes y el cauce la cual actúa reteniendo el caudal en épocas de crecidas extraordinarias. La fotografía c) muestra el arroyo en una creciente y la acción de la vegetación sobre el escurrimiento.

El último parámetro a analizar es la llanura de inundación (p3) ya que entre las múltiples funciones que tiene, regula los caudales de crecida reduciendo la energía y favoreciendo las conexiones ecológicas entre cauce y ribera. En el caso del tramo seleccionado la llanura de inundación presenta restricciones antropogénicas que afectan el comportamiento del agua durante las crecidas extraordinarias (fig. 4) Estas restricciones están en relación con la instalación de viviendas y un uso de suelo recreativo. 
Figura 4. Funcionalidad de la llanura de inundación
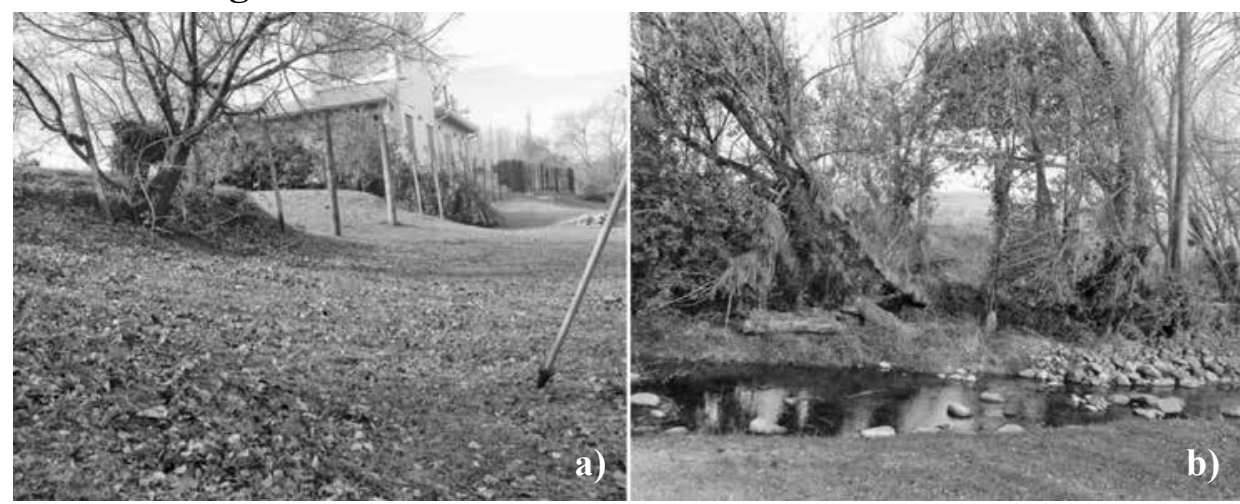

Fuente: Volonté (2014).

En la fotografía a) se observa las viviendas construidas sobre la llanura de inundación. En la fotografía b) se presenta un ejemplo de la vegetación introducida (Populus alba).

En la tabla 2 se detalla la puntuación obtenida para cada parámetro. El total de puntos alcanzado fue 27 lo cual indica una calidad hidrogeomorfológica muy buena.

Tabla 2. Valoración del Bloque I

\begin{tabular}{|l|l|c|}
\hline \multicolumn{1}{|c|}{ Parámetro } & \multicolumn{1}{|c|}{ Explicación } & Puntos \\
\hline $\begin{array}{l}\text { Naturalidad del régimen de } \\
\text { caudal }\end{array}$ & $\begin{array}{l}\text { La cantidad de caudal circulante responde a } \\
\text { la dinámica natural no siendo afectada por la } \\
\text { actividad antropogénica. }\end{array}$ & 10 \\
\hline $\begin{array}{l}\text { Disponibilidad y movilidad } \\
\text { de sedimentos }\end{array}$ & $\begin{array}{l}\text { No existen represas ni diques que retengan } \\
\text { sedimentos. Se observa indicios de } \\
\text { dificultades leves debido al crecimiento de } \\
\text { especies vegetales (-1). }\end{array}$ & 9 \\
\hline $\begin{array}{l}\text { Funcionalidad de la llanura } \\
\text { de inundación }\end{array}$ & $\begin{array}{l}\text { La llanura de inundación tiene obstáculos } \\
\text { transversales: vegetación introducida, } \\
\text { viviendas y un área recreativa (-2). }\end{array}$ & 8 \\
\hline \multicolumn{2}{|c|}{ Calidad Funcional del sistema: muy buena } & $\mathbf{2 7}$ \\
\hline
\end{tabular}

Fuente: elaborado por Antonela Volonté (2014) sobre la base de Ollero Ojeda (2009).

\section{Bloque II. Calidad del cauce}

La calidad del cauce es un elemento clave del sistema fluvial natural dado que es un indicador fundamental del estado del mismo. El cauce 
incluye la zona más deprimida dentro de la llanura que, por lo general, alberga las aguas concentradas. Corresponde a una franja del terreno bien establecida pero que su ocupación varía según el caudal, siendo mínima con los estiajes y máxima en las crecidas. El dominio del cauce también se extiende a la llanura de inundación. La calidad del cauce se obtiene a partir de la suma de las valoraciones de tres parámetros: (p1) naturalidad del trazado y de la morfología en planta; (p2) la continuidad, la naturalidad del lecho y los procesos longitudinales y verticales y (p3) la naturalidad de las márgenes y la movilidad lateral.

Con respecto a (p1) el cauce se encuentra modificado por la acción antropogénica debido a la realización de un canal artificial para atenuar los efectos de las crecidas ordinarias. Este canal se encuentra con agua durante la mayor parte del año (fig. 5). Es la única modificación que presenta el cauce y afecta a menos del $10 \%$ de la longitud del tramo.

En el segundo parámetro $(\mathrm{p} 2)$, si bien el cauce se mantiene natural y los procesos hidrológicos son funcionales, existen obstáculos, como el dique, que altera levemente el funcionamiento hidrológico; este dique embalsa menos del $25 \%$ de la longitud del sector. Con respecto a (p3) el cauce tiene la capacidad de movilizarse lateralmente ya que sus márgenes presentan una morfología natural. En este momento el cauce no presenta movilidad lateral porque se encuentra en una fase de profundización. El total de puntos alcanzados para la calidad del cauce fue 27 lo cual indica una calidad hidrogeomorfológica muy buena. 
Figura 5. Naturalidad del trazado y de la morfología en planta
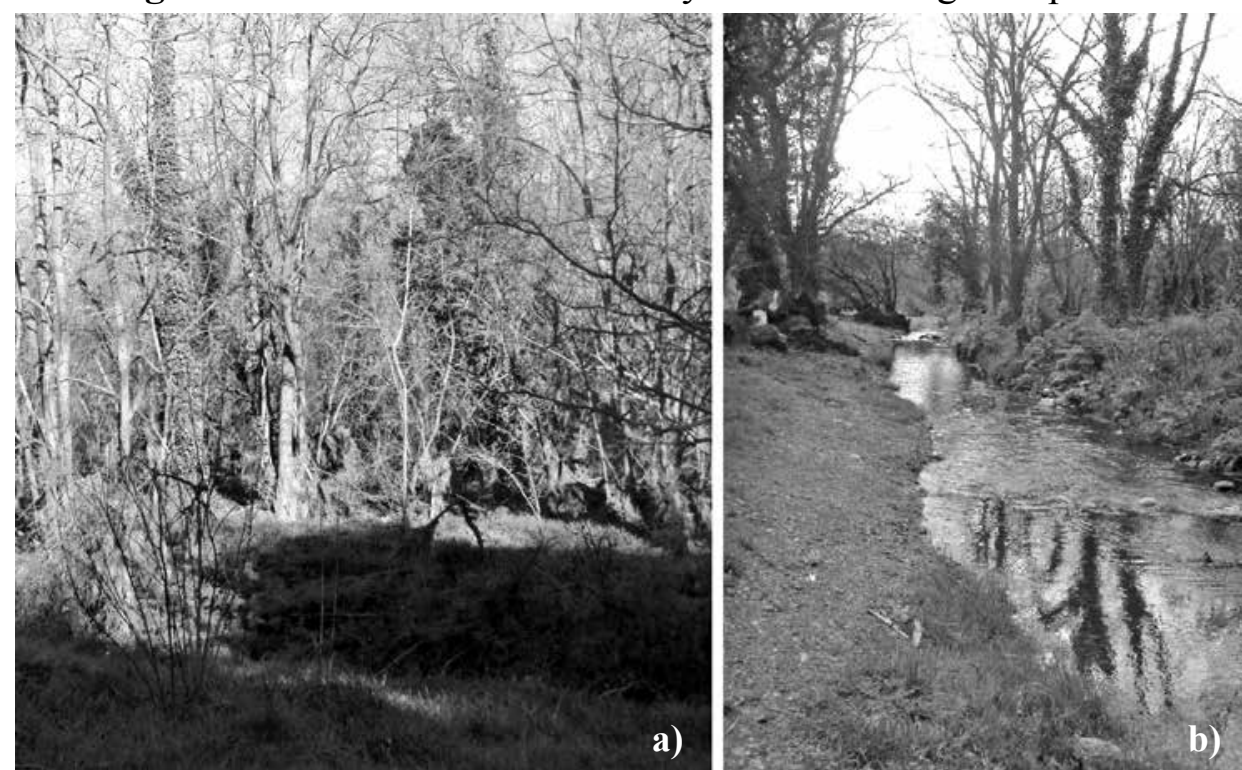

Fuente: Volonté, 2014.

En la fotografía a) se observa el canal artificial y el cauce natural. En la fotografía b) se observa el canal artificial.

En la tabla 3 se puede observar la puntuación obtenida para cada parámetro, la calidad del cauce es muy buena.

Tabla 3. Valoración del Bloque II

\begin{tabular}{|l|l|c|}
\hline \multicolumn{1}{|c|}{ Parámetro } & \multicolumn{1}{|c|}{ Explicación } & Puntos \\
\hline $\begin{array}{l}\text { Naturalidad del trazado y } \\
\text { de la morfología en planta }\end{array}$ & $\begin{array}{l}\text { El trazado se encuentra modificado } \\
\text { levemente por la presencia de un canal } \\
\text { artificial que no afecta en gran medida el } \\
\text { funcionamiento natural del sistema (-1) }\end{array}$ & 9 \\
\hline $\begin{array}{l}\text { Continuidad y naturalidad } \\
\text { del lecho y de los procesos } \\
\text { longitudinales y verticales }\end{array}$ & $\begin{array}{l}\text { En el sector funcional hay un dique que } \\
\text { interrumpe la continuidad del cauce, por lo } \\
\text { tanto se restan 2 puntos. }\end{array}$ & 8 \\
\hline $\begin{array}{l}\text { Naturalidad de las } \\
\text { márgenes y de la movilidad } \\
\text { lateral }\end{array}$ & $\begin{array}{l}\text { El cauce es natural y tiene la capacidad de } \\
\text { movilizarse lateralmente. }\end{array}$ & 10 \\
\hline \multicolumn{2}{|c|}{ Calidad del cauce: muy buena } & $\mathbf{2 7}$ \\
\hline
\end{tabular}

Fuente: elaborado por Antonela Volonté (2014) sobre la base de Ollero Ojeda (2009). 


\section{Bloque III. Calidad de las riberas}

La calidad de las riberas es el último de los bloques que se consideran para evaluar el sistema fluvial. Se tiene en cuenta 3 parámetros relacionados con la hidrogeomorfología del corredor ribereño: (p1) la continuidad longitudinal del corredor ribereño; (p2) el ancho y su calidad intrínseca y (p3) la estructura, naturalidad y la conectividad entre sus ambientes.

Con respecto a (p1) el corredor ribereño no es continuo a lo largo del tramo, sino que el mismo se ve perturbado por diferentes construcciones. Las discontinuidades suponen entre un 45 y $55 \%$ del área del tramo y son permanentes en más del $70 \%$. Sobre una de las márgenes se encuentran viviendas y sobre la otra, la parte posterior de un parque recreativo.

El parámetro (p2) se ve reducido por la ocupación humana. Se observa una ocupación creciente del espacio ribereño debido a la presencia de viviendas que han generado que la misma disminuya su ancho y pierda la continuidad, que es un valor aún más frágil. El ancho medio del corredor actual es superior al $80 \%$ del potencial. La ruptura de la continuidad longitudinal del corredor ribereño se debe al uso antropogénico.

Respecto a (p3) se conserva la estructura natural de las especies y predominan las herbáceas con una distribución continua. En trabajos previos se identificaron 12 especies herbáceas y 1 arbórea como las representativas del área de ribera. Las predominantes fueron: Cortaderia selloana y Cynodon dactylon mientras que en menor cantidad se encontraron Populus spp., Galium broterianum y Carduus thoermeri. En lo que concierne a la naturalidad (fig. 6) las especies se encuentran adaptadas al medio ribereño detectándose algunas invasoras. Existen diferentes usos de las riberas por parte de la población que genera alteraciones leves. 
http://dx.doi.org/10.15359/rgac.1-54.6

Antonela Volonté, Alicia M. Campo, Verónica Gil. Ecological conditions of the

San Bernardo stream's low basin, Sierra de la Ventana, Argentina

Figura 6. Riberas naturales

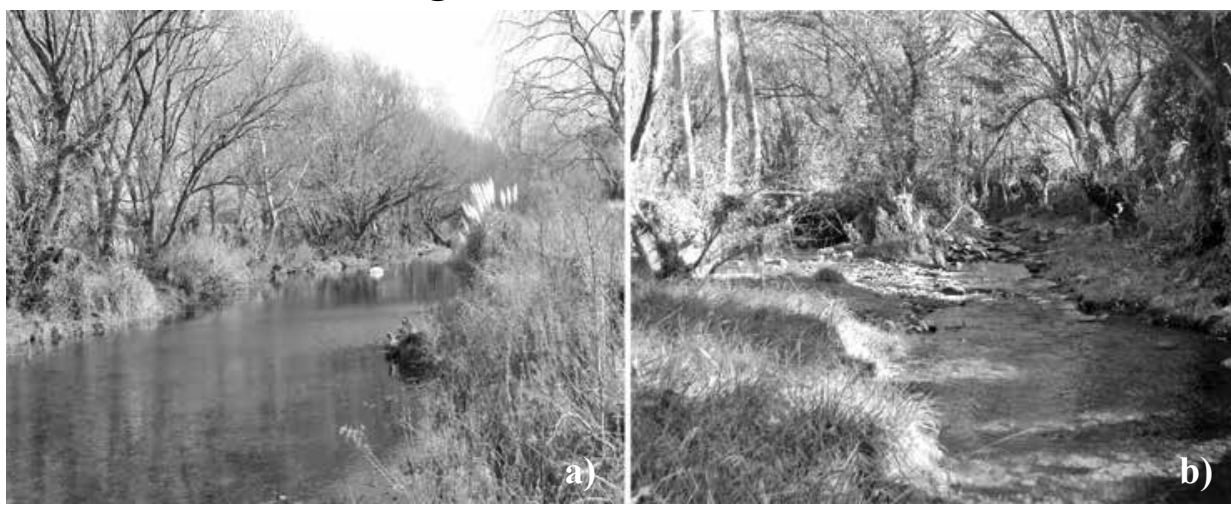

Fuente: Volonté (2014).

En la fotografía a) y b) se observan las riberas en estado natural.

En la tabla 4 se puede observar el total de puntos alcanzado en el Bloque III. Este bloque fue el que presentó el menor puntaje (19) y se encuentra con mayores transformaciones por lo que su calidad es moderada.

Tabla 4. Valoración del Bloque III

\begin{tabular}{|l|l|c|}
\hline \multicolumn{1}{|c|}{ Parámetro } & \multicolumn{1}{|c|}{ Explicación } & Puntos \\
\hline $\begin{array}{l}\text { Continuidad longitudinal } \\
\text { de las riberas }\end{array}$ & $\begin{array}{l}\text { La discontinuidad es debida a la presencia } \\
\text { de viviendas sobre la margen, lo cual supone } \\
\text { entre 45 \% y 55\% \% la longitud total de la } \\
\text { ribera del tramo (-6). }\end{array}$ & 4 \\
\hline $\begin{array}{l}\text { Anchura del corredor } \\
\text { ribereño }\end{array}$ & $\begin{array}{l}\text { El ancho de la ribera fue reducido por } \\
\text { ocupación antropogénica en un 20\% \% (-2). }\end{array}$ & 8 \\
\hline $\begin{array}{l}\text { Estructura, naturalidad y } \\
\text { conectividad transversal } \\
\text { del corredor ribereño }\end{array}$ & $\begin{array}{l}\text { Existe presión antropogénica sobre las } \\
\text { riberas, se observa recolección de madera } \\
\text { muerta y uso recreativo entre un 25 y 50\% } \\
\text { de la superficie de la ribera actual (-2). Con } \\
\text { respecto a la naturalidad de la vegetación } \\
\text { ribereña, se observan algunos ejemplares } \\
\text { introducidos (-1). }\end{array}$ & 7 \\
\hline \multicolumn{2}{|c|}{ Calidad de las riberas: moderada } & 19 \\
\hline
\end{tabular}

Fuente: elaborado por Volonté (2014) sobre la base de Ollero (2009).

A modo de síntesis en la tabla 5 se puede observar el resultado final. El total de puntos alcanzado fue 73 lo cual implica una calidad 
hidrogeomorfológica buena. La calidad funcional del sistema se considera muy buena ( 27 puntos) debido principalmente a la ausencia de regulación hidrológica al igual que la calidad del cauce (27 puntos). La calidad de las riberas fue la que obtuvo el menor puntaje (19), dando como resultado una calidad moderada debido a la acción antropogénica sobre las márgenes, la cual disminuye notablemente su funcionalidad.

Tabla 5. Valoración integral del Índice Hidrogeomorfológico

\begin{tabular}{|l|c|}
\hline \multicolumn{2}{|l|}{ Índice para la evaluación de la calidad hidrogeomorfológica de sistemas fluviales } \\
\hline Calidad funcional del sistema & 27 puntos \\
\hline Calidad del cauce & 27 puntos \\
\hline Calidad de las riberas & 19 puntos \\
\hline \multicolumn{1}{|c|}{ Total } & $\mathbf{7 3}$ puntos \\
\hline
\end{tabular}

Fuente: elaborado por Volonté (2014).

\section{Conclusión}

Los procesos geomorfológicos son la base fundamental del correcto funcionamiento de los cursos fluviales y los indicadores hidrogeomorfológicos son clave para determinar el estado ecológico de los ríos. El índice aplicado en este trabajo es una herramienta de valoración del estado ecológico.

La aplicación de IHG a un tramo del arroyo San Bernardo permitió obtener como resultado una valoración de la calidad buena. Las áreas más afectadas son las riberas dado que presentan una calidad menor (19 puntos) por la ocupación antropogénica en cambio tanto el cauce como el sistema en general se encuentran dentro de los límites de muy buena calidad (27 puntos).

El impacto más importante en este tramo fluvial es el producido por la instalación de viviendas sobre una de las márgenes ya que dificulta la dinámica lateral normal. La calidad hidrogeomorfológica se mejoraría alejando o eliminando los obstáculos como por ejemplo disminuyendo la instalación de viviendas y las actividades recreativas de la zona ocupada por el cauce y las orillas. El Índice Hidrogeomorfológico se continuará aplicando en distinto tramos del arroyo para obtener una valoración de todo el curso fluvial y en distintos afluentes para realizar comparaciones a los efectos de determinar el estado ecólogico de la cuenca en su totalidad. 


\section{Agradecimientos}

El presente trabajo se desarrolló en el marco de los siguientes proyectos:

Geografía Física aplicada al estudio de la interacción sociedad-naturaleza. Problemáticas a diferentes escalas témporo-espaciales. Dirigido por la Dra. Alicia María Campo. Secretaría de Ciencia y Tecnología, Universidad Nacional del Sur. amcampo@uns.edu.ar

Dinámica hidrogeomorfológica aplicada al estudio del peligro hidrometeorológico en cuencas serranas. Dirigido por la Dra. Verónica Gil. Consejo Nacional de Investigaciones Científicas y Técnicas. verogil@uns.edu.ar

\section{Referencias}

Campo de Ferreras, A., Capelli de Steffens, A. y Diez, P. (2004). El clima del suroeste bonaerense. Buenos Aires, Universidad Nacional del Sur. Campo, A., Volonté, A. y Gil, V. (2014). Evolución espacial en la morfometría de los sedimentos. Cuenca baja del arroyo San Bernardo. X Jornadas de Geografía Física, Malargüe (pp. 1-10).

García, J., Gómez, A. y Ortigosa, L. (1987). Aspectos dinámicos de un cauce fluvial en el contexto de su cuenca: el ejemplo del río Oja. Logroño: Instituto Pirenaico de Ecología, JACA e Instituto de Estudios riojanos (p. 113).

Gentili, J., Campo, A. y Gil, V. (2011). Distribución espacial y temporal de las precipitaciones en la cuenca alta del arroyo Sauce Corto, Sistema de Ventania. En: GAEA. Actas Científicas CNG $72^{\circ}$ Semana de Geografía. Mar del Plata, GAEA. (pp. 115-126).

Gil, V. (2009). Hidrogeomorfología de la cuenca alta del río Sauce Grande aplicada al peligro de crecidas. Tesis doctoral en Geografía. Universidad Nacional del Sur, Argentina (p. 269).

Harrington, H. (1947). Explicación de las hojas geológicas $33 m$ y 34m

Sierra de Curamalal y de la Ventana. Buenos Aires: Ministerio de Industria y Minería.

Matteucci, S. (2012). Ecorregión Pampa. En: Morello, J., Matteucci, S., Rodríguez, A. y Silva, M.: Ecorregiones y complejos ecosistémicos argentinos. Buenos Aires: Ed. Orientación Gráfica (pp. 391-446). 
Martínez, J., Correcher, E., Piñón, A., Martínez, A. y Pujante, M. (2004). Estudio del estado ecológico de los ríos de la cuenca hidrográfica del Júcar (España) mediante el índice BMWP. Revista Limnética, Asociación Española de Limnología, Madrid, Vol. 23 (3-4), (pp. 331-346).

Ollero, A. (2009). Aplicación del índice hidrogeomorfológico IHG en la cuenca del Ebro. Guía metodológica. Zaragoza, Ministerio de Medio Ambiente y Medio Rural y Marino.

Ordeix, M., Camprodom, J., y Guixe, D., (2012). "Metodología de diagnosis y evaluación del estado ecológico y la biodiversidad en restauraciones fluviales". Restauración y gestión ecológica fluvial. Ed. Centre Forestal Tecnòlogic de Catalunya (pp. 12-22).

Sellés, J. (2001). Geología de la Ventania. Provincia de Buenos Aires, Argentina. Journal of Iberian Geology, Vol. (27), (pp. 43-69)

Volonté, A., Duval, V., Gil, V. y Campo, A. (2013). Vegetación ribereña en la cuenca baja del arroyo San Bernardo, Sierra de la Ventana. Actas Congreso Internacional de Geografía. $74^{\circ}$ Semana de Geografía. Trevelín, Chubut (pp. 1-10).

Zapperi, P., Casado, A., Gil, V. y Campo, A. (2006). Caracterización de las precipitaciones invernales en el suroeste bonaerense. Buenos Aires: IV Jornadas Interdisciplinarias del Sudoeste Bonaerense. Universidad Nacional del Sur (pp. 1-9).

Zapperi, P., Ramos, M., Gil, V. y Campo, A. (2007). Caracterización de las precipitaciones estivales en el Suroeste bonaerense. Buenos Aires: Contribuciones Científicas, Sociedad Argentina de Estudios Geográficos, $68^{\circ}$ Semana de Geografía (pp. 1-12). 